\title{
BMJ Open Effectiveness of a tailored rehabilitation versus standard strengthening programme for patients with shoulder pain: a protocol for a feasibility randomised controlled trial (the Otago MASTER trial)
}

\author{
Daniel C Ribeiro, ${ }^{\circledR} 1$ Zohreh Jafarian Tangrood, ${ }^{\oplus 1}$ Gisela Sole, ${ }^{\oplus 1} \mathrm{~J} \mathrm{Haxby}$ Abbott $^{\odot 2}$
}

To cite: Ribeiro DC, Jafarian Tangrood Z, Sole G, et al. Effectiveness of a tailored rehabilitation versus standard strengthening programme for patients with shoulder pain: a protocol for a feasibility randomised controlled trial (the Otago MASTER trial). BMJ Open 2019;9:e028261. doi:10.1136/ bmjopen-2018-028261

- Prepublication history and additional material for this paper are available online. To view please visit the journal (http:// dx.doi.org/10.1136/bmjopen2018-028261).

Received 06 December 2018 Revised 06 July 2019 Accepted 09 July 2019

Check for updates

(C) Author(s) (or their employer(s)) 2019. Re-use permitted under CC BY-NC. No commercial re-use. See rights and permissions. Published by BMJ.

${ }^{1}$ School of Physiotherapy, University of Otago, Dunedin, New Zealand

${ }^{2}$ Department of Surgical Sciences, Dunedin School of Medicine, University of Otago, Dunedin, New Zealand

Correspondence to Dr Daniel C Ribeiro; daniel.ribeiro@otago.ac.nz

\section{ABSTRACT}

Introduction Exercise therapy is the treatment of choice for the management of patients with shoulder subacromial pain. However, we do not know whether a tailored rehabilitation programme is more effective than a standardised strengthening programme. The aim of this feasibility trial is to assess: (1) participant recruitment rate, (2) the proportion of participants enrolled from the total number screened, (3) adherence to the rehabilitation programme, (4) drop-out rates, (5) obtain estimates of adverse reactions to treatment, (6) obtain estimates of intervention effects in order to inform the sample size of the fully-powered randomised controlled trial, (7) conduct a preliminary cost-effectiveness analysis of the standardised strengthening and the tailored rehabilitation interventions.

Methods The MAnagement of Subacromial disorders of The shouldER (MASTER) trial, is a two-arm, patient-blinded and assessor-blinded, randomised controlled feasibility trial. Participants will be randomly allocated into one of the interventions group: tailored or standardised rehabilitation. To obtain estimates of intervention effects, we will compare changes in pain and shoulder-related disability scores between the two intervention groups using a repeated mixed-model analysis of variance, with alpha set at 0.05 , and power at $80 \%$. Since this is a feasibility study, we will not adjust alpha for multiple comparisons. To determine whether it is feasible to conduct the full trial, we will consider $75 \% \mathrm{Cl}$ as the probability threshold at 3-month follow-up.

Ethics and dissemination This study was approved by the University of Otago Ethics Committee (Ref: H17/080). Findings from this study will be presented at national and international conferences, and will be submitted for publication in a peer-reviewed journal.

Trial registration number ANZCTR: 12617001405303

\section{INTRODUCTION}

Shoulder pain is the third most common musculoskeletal complaint, with a 1-year prevalence of $18.1 \% .{ }^{1}$ Shoulder pain is associated
Strengths and limitations of this study

- This protocol will compare one intervention that is tailored to patient's physical impairments with a standardised strengthening programme.

- The feasibility trial will include economic evaluation, and implementation-based process evaluation of the intervention planned.

- Clinicians were not blinded to interventions due to nature of interventions, and that is a source of potential bias.

with high socioeconomic burden. ${ }^{2}$ In Sweden, the average annual cost of shoulder subacromial pain is estimated $\$ 4139$ per patient. $^{2}$ In New Zealand, a total of $\$ 134$ million was spent by Accident Compensation Corporation (ACC) in rehabilitation for shoulder injuries from 2005 to 2013 (\$14 million/year). ${ }^{3}$ Shoulder subacromial pain is a challenging disorder with slow recovery, ${ }^{4}$ with only $50 \%$ of new episodes presenting full recovery within 6 months. ${ }^{5}$

Exercise therapy is the first approach for the management of shoulder subacromial pain, and is recommended, for example, by the British Elbow \& Shoulder Society (BESS). ${ }^{6}$ A number of systematic reviews have been published in this area. ${ }^{7-13}$ Some reviews suggest exercise therapy and or manual therapy to be effective for improving pain and function in patients with shoulder pain, but highlight the limited strength of evidence to support this. ${ }^{7814}$ Three reviews reported exercise therapy to be more effective than control or placebo, ${ }^{8} 1011$ while a Cochrane Review reported no clinically important difference between manual therapy and exercise vs placebo. ${ }^{9}$ The addition of manual 
therapy to exercise has been supported in incremental effects trials ${ }^{15-17}$ and by another recent systematic review. ${ }^{10}$ Optimal treatment strategies are needed to improve treatment effect, speed recovery and decrease shoulder pain recurrence. There are uncertainties regarding which types of exercise are more effective and cost-effective for the management of patients with shoulder subacromial pain. ${ }^{101819}$ In addition, there are competing approaches of exercise regimen (eg, specific exercise, ${ }^{20}$ general strengthening exercise), with limited number of head-tohead trials comparing different combinations of exercise therapy and manual therapy interventions. ${ }^{910}$

The lack of data about process evaluation of previous trials hinder our ability to identify whether tested interventions failed to improve clinical outcomes due to being ineffective or poorly implemented. ${ }^{21}$ To address this, it is recommended trials to include process evaluation alongside the outcome evaluation, ideally from Phase II to Phase IV. ${ }^{21}$ Process evaluation studies provide valuable information regarding how, what and why interventions were delivered to patients during the trial, ${ }^{21-24}$ and help to understand why an intervention achieved (or not) its expected clinical outcomes. ${ }^{25}$ Such information is valuable for a number of stakeholders (eg, clinicians, government and policy-maker agencies) and improve translation of findings from trials to clinical practice. ${ }^{21}$ Hence, future trials on the management of shoulder subacromial pain should include process evaluation ${ }^{21}$ and economic evaluation ${ }^{10}$ conducted alongside the outcome evaluation. In addition, it has been recommended that future trials assessing the effect of novel exercise therapy programme to be compared with a valid placebo intervention. ${ }^{9}$

Shoulder subacromial pain is a complex disorder, with psychological factors and physical impairments influencing clinical outcomes. ${ }^{26-29}$ Pain beliefs seem to be associated with course of pain and disability, but the current level of evidence is low. ${ }^{29}$ A longitudinal prospective cohort study reported that psychosocial factors are associated with clinical outcomes, ${ }^{28}$ while a secondary analysis of a trial found that fear-avoidance beliefs contribute significantly to baseline disability but not to disability change scores after 3-month follow-up. ${ }^{26}$ Psychosocial factors and pain beliefs seem to play a role on clinical outcomes. Further longitudinal studies are still required to clarify which psychological factors can be targeted by treatment and whether modifying these psychological factors impact on clinical outcomes (ie, disability and pain) in patients with shoulder subacromial disorders. ${ }^{26} 272930$

Clinical examination is commonly performed by clinicians $^{3132}$ to inform management of patients with shoulder pain. Findings suggestive of structural diagnosis have inconsistent association with clinical outcomes ${ }^{30}$ and the diagnostic accuracy of orthopaedic tests is very limited. ${ }^{33}$ Hence, it has been suggested clinicians to perform a thorough clinical examination when assessing patients with shoulder disorders. ${ }^{31} 3334$

The role of scapular and shoulder muscle recruitment pattern and clinical outcomes is unclear. ${ }^{35}$ The report from the "Scapular Summit" suggests a potential role of scapular movement pattern on shoulder disorders, but highlighted it is currently not clear whether there is a causative effect between movement pattern and shoulder disorders. ${ }^{36}$ A recent study reported increased risk of shoulder pain among asymptomatic individuals, suggesting movement pattern might play a role on shoulder symptoms,${ }^{37}$ while a recent review found some preliminary evidence supporting the use of scapula-focused exercises when managing patients with shoulder pain, but it is unclear whether these exercises have any impact on pain and disability outcomes. ${ }^{35}$

Patients with shoulder pain present with physical impairments, and altered scapular and shoulder muscle recruitment patterns, ${ }^{38} 39$ these impairments are a potential target for therapeutic intervention. ${ }^{40}$ For example, patients with shoulder subacromial pain may show altered coordination between lower trapezius and serratus anterior, and the upper trapezius and lower trapezius during arm elevation task, ${ }^{39}$ and patients with symptomatic rotator cuff tear may show increased activity of latissimus dorsi when compared with healthy controls. ${ }^{38}$ Due to the variability of such altered muscle patterns, it is recommended that rehabilitation should tailor specific muscle and joint impairments presented by the patient ${ }^{31}$ and restore shoulder movement pattern. ${ }^{34}$ Further, preliminary evidence suggests that sustained shoulder mobilisation may reduce pain and improve range of motion in patients with shoulder subacromial pain, compared with sham sustained mobilisation. ${ }^{41}$ Trials of the effect of sustained glide and exercise on the management of other musculotendinous disorders (eg, tennis elbow) have found this intervention to be more effective than corticosteroid injection and wait-and-see. ${ }^{42}$

Laboratory-based studies suggest that: (1) clinician-administered sustained shoulder mobilisation offloads shoulder muscles, providing mechanical support to the shoulder $^{43}$; (2) patient-administered sustained shoulder mobilisation leads to similar changes in muscle activity levels as clinician-administered mobilisation, supporting the use of home-based mobilisation for shoulder rehabilitation $^{44}$; (3) patients with shoulder pain present immediate reduction in pain levels, increased range of motion and altered muscle activity levels in response to sustained shoulder mobilisation. ${ }^{45}$ These findings, and anecdotal evidence from clinical practice, suggest that sustained mobilisation temporarily changes the control of scapular and shoulder muscles. Such temporary change might give the clinician a therapeutic window to strengthen muscles with less pain while keeping a better control of scapular and shoulder muscles.

It is unclear whether a tailored rehabilitation (combining sustained mobilisation with specific motor control exercises) is more effective than standardised exercise for shoulder pain patients. Tailored rehabilitation focuses on each patient's specific impairments and some studies found preliminary findings that a tailored programme is effective for managing patients 
with shoulder pain. ${ }^{20} 46-49$ An alternative to a tailored programme is a standardised shoulder rehabilitation that adopts a more generic approach, with standardised stretching and strengthening exercises being prescribed for all patients, and may also be delivered in small group sessions, reducing the cost of the physiotherapy session. It is unclear which approach leads to better clinical outcomes and is more cost-effective.

Efficacy trials are designed to test if an intervention works under the ideal circumstances. ${ }^{50}$ This type of trial maximises the probability of observing the effect of an intervention (assuming such effects exist), and prioritise internal validity of the study design.$^{50}$ In efficacy trials, the intervention is standardised, delivered under an ideal setting, with highly trained clinicians. ${ }^{50}$ It is recommended for efficacy trials to incorporate economic evaluation (ie, cost-efficacy) alongside the clinical efficacy assessment. ${ }^{51}$ By assessing clinical and cost-efficacy it is possible to determine whether an intervention is likely to be efficacious for a group of patients, and whether delivering the health outcomes are likely to be good value for money. ${ }^{52}$ Ideally, clinical and cost-efficacy studies should be conducted prior to clinical-effectiveness and cost-effectiveness pragmatic trials. ${ }^{51}$

The aim of our full study is to assess the clinical-efficacy and cost-efficacy of tailored rehabilitation programme for the treatment of shoulder subacromial pain. Prior to conducting a fully-powered randomised controlled trial (RCT), we propose an efficacy feasibility trial aiming to assess: (1) participant recruitment rate, (2) the proportion of participants enrolled from the total number screened, (3) adherence to the rehabilitation programme, (4) drop-out rates, (5) obtain exploratory estimates of adverse events, (6) obtain exploratory estimates of intervention effects in order to inform the sample size of the fully-powered RCT, (7) conduct a preliminary cost-effectiveness analysis of the standardised strengthening and the tailored rehabilitation interventions.

\section{METHODS \\ Design}

The Management of subacromial disorders of the shoulder (MASTER) trial, is a two-arm, patient-blinded and assessor-blinded, feasibility RCT. Participants will be randomly allocated into one of the interventions group: tailored rehabilitation or standardised rehabilitation (figure 1).

When preparing this protocol, we followed the Standard Protocol Items: Recommendations for Interventional Trials statement, ${ }^{53}$ and the template for intervention description and replication (TIDieR) checklist and guide. ${ }^{54}$ When reporting the feasibility trial, we will follow the Consolidated Standards of Reporting

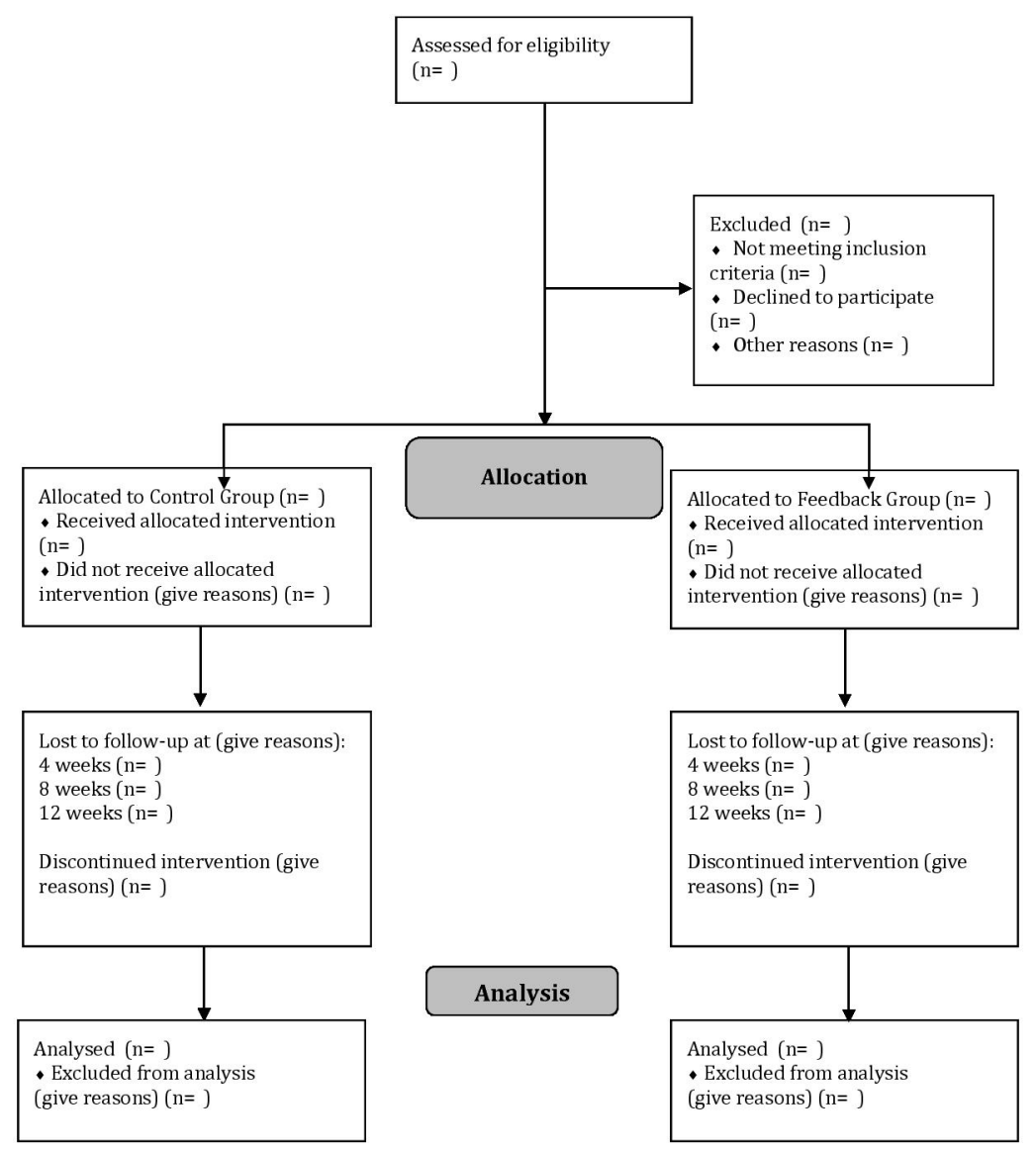

Figure 1 Diagram of participant flow at the Otago MAnagement of Subacromial disorders of The shouldER (MASTER) feasibility trial. 
Table 1 WHO trial registration data set

\begin{tabular}{|c|c|}
\hline Data category & Information \\
\hline $\begin{array}{l}\text { Primary registry and trial identifying } \\
\text { number }\end{array}$ & ACTRN 12617001405303 \\
\hline Date of registration in primary registry & 04/10/2017 \\
\hline $\begin{array}{l}\text { Source of monetary or material } \\
\text { support }\end{array}$ & Health Research Council of New Zealand Feasibility Grant (17/536) \\
\hline Primary sponsor & University of Otago \\
\hline Contact for public queries & daniel.ribeiro@otago.ac.nz \\
\hline Contact for scientific queries & Dr Daniel Cury Ribeiro, School of Physiotherapy - University of Otago \\
\hline Public title & $\begin{array}{l}\text { Tailored vs standard strengthening rehabilitation for patients with shoulder pain: a } \\
\text { feasibility trial }\end{array}$ \\
\hline Scientific title & $\begin{array}{l}\text { The effectiveness of a tailored rehabilitation vs standard strengthening programme for } \\
\text { patients with shoulder pain: a feasibility randomised controlled trial (the Otago MASTER } \\
\text { trial) }\end{array}$ \\
\hline Country of recruitment & New Zealand \\
\hline Health condition or problem studied & Shoulder subacromial pain \\
\hline Interventions & Tailored and standardised strengthening exercise \\
\hline Key inclusion and exclusion criteria & Adult healthcare workers (from 18 to 65 years old), with subacromial shoulder pain. \\
\hline Study type & Interventional \\
\hline Date of first enrolment & $12 / 02 / 2018$ \\
\hline Target sample size & 25 \\
\hline Recruitment status & Recruiting \\
\hline Primary outcome & $\begin{array}{l}\text { (1) Recruitment rate, (2) Proportion of participants enrolled from the total number } \\
\text { screened and (3) Adherence to the rehabilitation programme. }\end{array}$ \\
\hline Key secondary outcome & $\begin{array}{l}\text { (1) Drop-out rates, (2) pain level, (3) Shoulder-Related Disability-Patient Specific } \\
\text { Functional Scale, (4) quality-adjusted life year, (5) Shoulder Pain and Disability Index, (6) } \\
\text { Pain Self-Efficacy Questionnaire; (7) adverse reactions. }\end{array}$ \\
\hline
\end{tabular}

MASTER, Management of subacromial disorders of the shoulder.

Trials statement for non-pharmacological treatment. ${ }^{55}$ WHO trial registration data set information is described in table 1.

\section{Participants}

Participants with shoulder subacromial pain will be recruited to take part in the study.

\section{Inclusion and exclusion criteria}

Participants from 18 to 65 years old, with mechanical shoulder pain will be recruited to participate in the study. Participants will be screened as per the BESS guidelines. ${ }^{56}$ The process recommended by the BESS guidelines screens for: red flags (eg, tumour, unreduced dislocation, acute rotator cuff tear, infection), shoulder pain arising from the cervical spine, the shoulder instability, acromioclavicular joint disease and adhesive capsulitis. ${ }^{56}$

Participants will be included if they present one positive finding on the following tests: (1) painful arc movement during shoulder flexion or abduction, (2) Jobe's test $^{56}$ or (3) pain on resisted lateral rotation or abduction. ${ }^{57}$ Given the limited evidence from clinical tests for diagnosing patients with shoulder subacromial pain, ${ }^{33}$ we opted to widen the criteria proposed by BESS and add criteria \#3. We include two additional tests (resisted lateral rotation and shoulder abduction) ${ }^{57} \mathrm{~A}$ previous study reported pain on external rotation has $34.5 \%$ of sensitivity, $100 \%$ specificity, $42 \%$ accuracy for identifying any degree of subacromial disorder. ${ }^{57}$ Pain on shoulder abduction presented $55 \%$ of sensitivity, $75 \%$ specificity, $57 \%$ accuracy and a likelihood ratio of $2.2 \%$ for identifying any degree of subacromial disorder. In addition, pain on external rotation was the most accurate test for identifying partial-thickness tear. ${ }^{33} 57$

We will exclude participants with the history of shoulder dislocation, shoulder subluxation, shoulder surgery and cervical surgery within the last 6 months, ${ }^{58}$ participants with any kind of symptoms of systematic inflammation or disease, signs of paraesthesia in the upper extremities, hemiplegic shoulder pain, frozen shoulder or positive clinical signs of full thickness rotator cuff tear ${ }^{59}$ will be excluded.

\section{Sample size}

Being a feasibility RCT, the present study is not designed to assess the efficacy of the experimental intervention. ${ }^{6061}$ We estimated the sample size based on expected 
characteristics of the full trial. ${ }^{62}$ Based on recommendations by Whitehead et $a l^{62}$ the sample size of a feasibility study should be estimated based on the expected range for the effect size, the power and alpha (both established a priori), and the total number of arms of treatment planned for the full trial.

Whitehead et $a l^{62}$ recommend the sample sizeto be based on ranges of effect sizes (ie, extra small, small, medium and large). Therefore, to estimate the sample size for the feasibility trial, we used the Shoulder Pain and Disability Index (SPADI) as primary outcome measure; we assumed a minimum clinically important difference of 8 points $^{63}$ and a SD of 24 points on SPADI total score. ${ }^{63}$ This represents a standardised effect size of 0.3 . For estimating the sample size, we set power at $80 \%$, two-tailed between-group comparison, with alpha at 0.05. Therefore, the minimum sample size for this feasibility RCT is 10 participants per arm of treatment. ${ }^{62}$ Assuming a 20\% loss to follow-up, ${ }^{64}$ a total sample size of 25 participants is required.

\section{Recruitment}

Recruitment will take place in Dunedin, New Zealand. Participants will be recruited through general practitioners and hospitals and newspaper advertisements. In previous studies, we have successfully recruited participants with these methods of recruitment. Participants will be screened by a physiotherapist with more than 5 years of clinical experience, and with a postgraduate qualification in Musculoskeletal or Sports Physiotherapy (or related field).

\section{Informed consent and baseline assessment}

Once participants are assessed for eligibility, a clinical researcher will seek informed consent from participants. Participants may consent to take part in the study after screening or few days later, if they request time for considering taking part in the study. Participants will be asked to complete the baseline assessments and questionnaires for recording demographic data (age, height, weight), and baseline measurements for the primary and secondary outcomes.

\section{Randomisation}

Participants will be individually randomly allocated (1:1 ratio) into one of the intervention groups (ie, tailored physiotherapy or standardised physiotherapy). The randomisation schedule will be computer-generated by a research administrator, and concealed in numbered sealed and opaque envelopes. A research administrator will provide the envelope to the clinician delivering the interventions.

\section{Blinding}

Participants will be blinded to interventions. Outcome assessors will be blinded to group allocation. Clinicians delivering the interventions will not be blinded to group allocations due to nature of intervention.

\section{Procedures}

Experienced clinicians will deliver interventions for both groups. Clinicians will have a postgraduate diploma in musculoskeletal rehabilitation (or related field) and a minimum of 5 years of clinical experience. Outcome measures will be assessed by a physiotherapist who is blinded to group allocation. We will run a minimum of four training sessions lasting for 1 hour with clinicians to ensure they are familiarised with the protocol. To ensure they are confident in delivering the planned interventions, we will provide them with manuals containing a detailed description of the planned intervention and will meet with them during the study to clarify any questions or concerns that may arise.

\section{Interventions}

Both groups will receive 16 individual, face-to-face sessions, each lasting for $60 \mathrm{~min}$, twice per week, over an 8 -week period. This number and duration has been shown to improve clinical outcomes in patients with shoulder subacromial pain. ${ }^{65}$ Eight-week intervention period has been suggested as the minimum required to lead to improvement in pain and range of motion in patients with shoulder pain. ${ }^{65}$ The tailored and standardised rehabilitation interventions are described on tables 2 and 3, respectively. These descriptions were prepared following the TIDieR checklist and guide. ${ }^{54}$

Both groups will receive similar dosage of exercises. Participants will perform a total of 8 exercises per session of treatment, plus three stretches (control group) or up to three manual therapy techniques (tailored group). To ensure optimal internal validity of the trial, dosage of exercises for each group are planned to be equivalent. Details of tailored and standardised interventions are described on the Exercise Description Forms (online supplementary material 1 and 2 , respectively). The intensity of strengthening exercises will be monitored using a modified Borg scale. ${ }^{66}$ Rate of perceived exertion was shown to be valid for monitoring intensity of resistance training, ${ }^{67}$ and has been used in a previous trial for monitoring exercise intensity. ${ }^{68}$

Tailored rehabilitation: participants allocated to the tailored rehabilitation group will receive sustained mobilisation followed by exercises focusing on restoring normal movement pattern and the dynamic stability of the scapulothoracic and glenohumeral joints. ${ }^{31} 69$ The intervention will involve manual therapy techniques focusing on restoring the shoulder and scapular movement to reduce pain, ${ }^{70}$ and motor control and progressive resistance training of impaired muscles. ${ }^{65} 69$

Standardised rehabilitation: participants allocated to this group will receive a progressive resistance training for all scapular and shoulder muscles and stretching exercise programme. ${ }^{71}$ This intervention focuses on restoring muscle flexibility and strength and has been shown to be more effective than 'no intervention' or control for reducing pain and disability. ${ }^{71}$ 
Table 2 Description of tailored rehabilitation intervention, as per the template for intervention description and replication (TIDieR) guide

\begin{tabular}{ll}
\hline Item number & Item \\
\hline 1. & $\begin{array}{l}\text { BRIEF NAME } \\
\text { Tailored rehabilitation }\end{array}$ \\
2. & WHY Describe any rationale, theory or goal of \\
\hline
\end{tabular}

2. WHY Describe any rationale, theory or goal of the elements essential to the intervention.

3.

WHAT

Materials: Describe any physical or informationa materials used in the intervention, including those provided to participants or used in intervention delivery or in training of intervention providers. Provide information on where the materials can be accessed (eg, online appendix URL).

4.

Procedures: Describe each of the procedures, activities and/or processes used in the intervention, including any enabling or support

\section{WHO PROVIDED}

For each category of intervention provider (eg, psychologist, nursing assistant), describe their expertise, background and any specific training given.

\section{$6 . \quad$ HOW}

Describe the modes of delivery (eg, face-to-face or by some other mechanism, such as internet or telephone) of the intervention and whether it was provided individually or in a group.

\section{$7 . \quad$ WHERE}

Describe the type(s) of location(s) where the intervention occurred, including any necessary infrastructure or relevant features.

8. activities.

\section{Description}

The tailored rehabilitation programme will focus on specific impairments presented by the patient. This intervention will consist of mobilisation with movement, passive accessory mobilisation, specific motor control exercises and specific muscle strengthening exercises. The tailored rehabilitation programme might be more effective than a standardised strengthening programme for patients with subacromial shoulder pain.

The tailored rehabilitation group will receive manual therapy techniques (including mobilisation with movement with taping), ${ }^{709}$ motor control and strengthening exercises. Manual therapy interventions delivered by the clinician might be performed with a belt. Motor control and strengthening exercises might be performed with the use of elastic bands or dumbbells. Home-based exercises will consist of self-mobilisation techniques that is performed with a belt. Detailed information is described on online supplementary file 1 .

Clinicians will choose exercises based on physical impairments presented during the physical assessment.

Experienced clinicians will deliver interventions for the tailored group. Clinicians will have a postgraduate diploma in musculoskeletal rehabilitation (or related field) and a minimum of 5 years of clinical experience. All clinicians will undergo a trial-specific training programme to ensure they understand the protocol and the rationale of the intervention. Clinicians will receive a detailed manual with information regarding the trial intervention.

Participants will receive individual, face-to-face sessions.

Interventions will be delivered in a private clinical practice.

Participants will receive 16 sessions, each lasting for a maximum of $60 \mathrm{~min}$, twice per week, over an 8-week period.

The exercise programme will comprise eight exercises plus three optional Describe the number of times the intervention was delivered and over what period of time including the number of sessions, their schedule and their duration, intensity or dose. manual therapy techniques (one for the cervical spine, one for the thoracic spine and one for the shoulder). Clinicians will decide on which technique to use based on participants' clinical presentation. The manual therapy techniques might consist of passive joint mobilisations (grade -IV, IV or +IV) or manipulation (for the cervical or thoracic spine).

Mobilisation with movement techniques will count as one of the 8 possible exercises to be performed within a session. This technique will be performed with 3 sets of 10 repetitions, with 30 s of rest between each set. Passive joint mobilisations will be performed with the following dosage: 3 sets, 30 s duration. Grade: -III or -IV will be performed if pain is dominant (as per physical assessment) or grade +III or +IV if stiffness dominant (as per physical assessment).

Joint manipulation will be performed once per session, if required, as per physical assessment. The clinician will have the freedom to decide which technique to perform.

Isometric exercises will be delivered with the following dosage: 2 sets, 10 repetitions, with $10 \mathrm{~s}$ hold each repetition. The isometric exercises will be progressed in two stages. The first stage will have the following dosage: 3 sets, 10 repetitions, with 10 s hold each repetition. The second stage will have the following dosage: 3 sets, 10 repetitions, with 20 s hold each repetition. There will be 10 s rest between repetitions, and 30 s rest between sets. 
Table 2 Continued

Item number Item Description

Dynamic strengthening exercises will be delivered with the following dosage: 2 sets of 10 repetitions. The dynamic strengthening exercises will be progressed in two stages. The first stage will have the following dosage: 3 sets of 10 repetitions. The second stage will have the following dosage: 3 sets of 20 repetitions.

All exercises should initially be performed in slow and controlled pace. All motor control exercise should initially be of low intensity and then progressed as described. Clinicians can increase dosage (repetitions, sets, or load) if the participant is able to perform low intensity exercise for two consecutive sessions.

The load for strengthening exercises will be determined through using the 10-point Rate of Perceived Exertion (RPE) scale, considering the affected side. Low intensity will be defined as 3-4 RPE, moderate intensity as 5-6 RPE, and high intensity as 7-8 RPE.Exercises will start with low intensity, and can progress to moderate and high intensity during the course of treatment.

\begin{tabular}{|c|c|c|}
\hline 9. & $\begin{array}{l}\text { TAILORING } \\
\text { If the intervention was planned to be } \\
\text { personalised, titrated or adapted, then describe } \\
\text { what, why, when and how. }\end{array}$ & $\begin{array}{l}\text { Interventions will be tailored based on physical assessment. } \\
\text { Participants will receive: } \\
\text { Shoulder mobilisation with movement if, during assessment, } \\
\text { participants improve range of motion and pain with the mobilization } \\
\text { with movement (MWM) technique. As part of the treatment, clinicians } \\
\text { might use an MWM taping technique. }{ }^{70} 95 \\
\text { Passive mobilisation on the cervical, thoracic spine or shoulder } \\
\text { (glenohumeral joint). These techniques will be performed if, during } \\
\text { assessment, participants present with stiffness or pain on passive } \\
\text { accessory movement at the cervical, thoracic spine or glenohumeral } \\
\text { joint. } \\
\text { Motor control exercises if, during assessment, participants present } \\
\text { with poor control of a specific muscle (eg, scapular control exercises, } \\
\text { dynamic control of glenohumeral joint). }{ }^{4096-98} \\
\text { Strengthening exercises if, during assessment, participants present with } \\
\text { muscle weakness. }{ }^{40}\end{array}$ \\
\hline 10. & $\begin{array}{l}\text { MODIFICATIONS } \\
\text { If the intervention was modified during the } \\
\text { course of the study, describe the changes (what, } \\
\text { why, when and how). }\end{array}$ & Not applicable. This is a protocol. \\
\hline 12. & $\begin{array}{l}\text { Actual: If intervention adherence or fidelity was } \\
\text { assessed, describe the extent to which the } \\
\text { intervention was delivered as planned. }\end{array}$ & Not applicable. This is a protocol. \\
\hline
\end{tabular}

\section{Concomitant care}

Participants may seek other healthcare services, if they wish to do so. In that case, we will ask them to record which healthcare services they accessed on a logbook while enrolled on the trial.

\section{Criteria for modifying or discontinuing the exercises}

Pain levels, as subjectively reported by participants, will be used for determining whether an exercise must be modified or discontinued. ${ }^{72}$ For this purpose of this study, we adopted a criteria used in a previous study. ${ }^{74}$ Participants will be encouraged to continue with an exercise as long as the reported pain levels ranging from slight to endurable. Participants should discontinue exercise or reduce load if: (1) pain increases beyond what is acceptable/bearable for the participant; (2) participant reports an immediate increase of pain by three points (on the numeric pain rating scale) during exercise; (3) pain persists longer than $30 \mathrm{~s}$ after completion of exercise; (4) an exercise cannot be performed due to pain, clinicians will be asked not to include that specific exercise for the next two sessions and replace that exercise another exercise (for the tailored rehabilitation group) or with a longitudinal traction or pendulum exercise (for the standardised rehabilitation group). ${ }^{8}$ All participants will receive homebased stretching and strengthening exercises, and will be asked to perform these once a day. 
Table 3 Description of standardised rehabilitation intervention, as per the template for intervention description and replication guide

Item number Item Description

BRIEF NAME
Standardised rehabilitation

2.

WHY Describe any rationale, theory or goal of The standardised rehabilitation intervention will focus on the elements essential to the intervention. strengthening of scapular and shoulder muscles. Strengthening exercise were shown to improve pain and disability in participants with subacromial shoulder pain.

\section{WHAT}

Materials: Describe any physical or informational materials used in the intervention, including those provided to participants or used in intervention delivery or in training of intervention providers. Provide information on where the materials can be accessed (eg, online appendix, URL).
The standardised rehabilitation group will receive strengthening exercises. These exercises might be performed with the use of elastic bands or dumbbells. Stretching exercise for the thoracic spine will be done using a foam roller. Two home-based exercises (resisted internal and external rotation of the humerus) will be performed using an elastic band. Detailed information is described on online supplementary file 2 .

Participants will start with 8 'core' strengthening exercises and three stretches. The clinician can replace one core strengthening activities and/or processes used in the intervention, including any enabling or support exercises by another strengthening exercises from a list of activities.

5. WHO PROVIDED
For each category of intervention provider
(eg, psychologist, nursing assistant), describe
their expertise, background and any specific
training given.

\section{HOW}

Describe the modes of delivery (eg, face-toface or by some other mechanism, such as internet or telephone) of the intervention and whether it was provided individually or in a group.

7. WHERE
Describe the type(s) of location(s) where
the intervention occurred, including any
necessary infrastructure or relevant features.
WHEN and HOW MUCH
Describe the number of times the intervention
was delivered and over what period of time
including the number of sessions, their
schedule and their duration, intensity or dose.

Interventions will be delivered in a private clinical practice.

Participants will receive 16 sessions, each lasting for a Experienced clinicians will deliver interventions for the standardised rehabilitation group. Clinicians will have a postgraduate diploma in musculoskeletal rehabilitation (or related field) and a minimum of 5 years of clinical experience. All clinicians will undergo a trial-specific training programme to ensure they understand the protocol and the rationale of the intervention. Clinicians will receive a detailed manual with information regarding the trial intervention.

Participants will receive individual, face-to-face sessions.

maximum of $60 \mathrm{~min}$, twice per week, over an 8-week period. The standardised rehabilitation will comprise 8 exercises plus three stretching exercise (one for the cervical spine, one for the thoracic spine and one for the shoulder).

Strengthening exercises will be delivered with the following dosage: 2 sets of 10 repetitions. The strengthening exercises will be progressed in three stages. The first will have the following dosage: 3 sets of 10 repetitions. The second stage will have the following dosage: 3 sets of 20 repetitions. For the third stage of progression, clinicians can choose to increase the load to moderate (based on RPE-see below) or replace the exercise by another one from the additional list.

All the exercises should initially be performed in slow and controlled pace.

The load for strengthening exercises will be determined through using the 10-point Rate of Perceived Exertion (RPE) scale, considering the affected side. Low intensity will be defined as 3-4 RPE, moderate intensity as 5-6 RPE and high intensity as 7-8 RPE. 


\section{Table 3 Continued}

\begin{tabular}{|c|c|c|}
\hline Item number & Item & Description \\
\hline 10. & $\begin{array}{l}\text { MODIFICATIONS } \\
\text { If the intervention was modified during the } \\
\text { course of the study, describe the changes } \\
\text { (what, why, when and how). }\end{array}$ & Not applicable. This is a protocol. \\
\hline 11. & $\begin{array}{l}\text { HOW WELL } \\
\text { Planned: If intervention adherence or fidelity } \\
\text { was assessed, describe how and by whom } \\
\text { and if any strategies were used to maintain or } \\
\text { improve fidelity, describe them. }\end{array}$ & $\begin{array}{l}\text { Participants' adherence to protocol will be assessed by } \\
\text { quantifying the number of home-based exercises performed. It } \\
\text { will be expressed as percentage of the total number of sessions } \\
\text { that should be been performed. } \\
\text { Clinician's adherence to protocol will be assessed by } \\
\text { quantifying the number of exercises and progressions that were } \\
\text { performed according to the protocol. This will be done through } \\
\text { audits of clinical notes, and will be expressed as percentage } \\
\text { of the total number of exercises and progressions that were } \\
\text { performed during the course of treatment. }\end{array}$ \\
\hline 12. & $\begin{array}{l}\text { Actual: If intervention adherence or fidelity } \\
\text { was assessed, describe the extent to which } \\
\text { the intervention was delivered as planned. }\end{array}$ & Not applicable. This is a protocol. \\
\hline
\end{tabular}

\section{Primary outcome measures}

The primary feasibility outcome measures are (figure 2):

1. Participant's recruitment rate, measured as number of participants per month.

2. Proportion of participants enrolled from the total number screened, expressed as the ratio 'number of enrolled participants/total number of screened participants', with reasons.

3. Adherence to the rehabilitation programme, measured as number of sessions attended, and expressed as a percentage of the total number of sessions.

4. Drop-out rates, measured as the number of participants who dropped-out, and expressed as a percentage of the total number of participants enrolled in the study.

\section{Secondary outcome measures}

The patient-reported outcome measures intended as the primary and secondary outcomes in the main trial will be used as secondary outcome measures in this feasibility trial (figure 2). These are: pain level as measured by a numeric pain scale ${ }^{75}$ and shoulder-related disability assessed using the 'Patient Specific Functional Scale' (PSFS), the SPADI ${ }^{76}$ and the pain self-efficacy questionnaire. ${ }^{77}$ The minimal clinically important difference for the numeric pain scale is one point, ${ }^{78}$ and for the PSFS is 1.3 (for small changes), 2.3 (for medium changes) and 2.7 (large changes). ${ }^{79}$ Although this feasibility trial is not powered to detect superiority, we will assess the magnitude of mean treatment effects for pain and physical function in relation to clinically important change, to inform the choice of primary outcome and sample size calculation for the main trial. Findings from this feasibility trial will help us selecting the primary outcome measure for the full trial. ${ }^{80}$

We will assess safety by recording all adverse events, both related and unrelated to interventions, in each group. The literature suggests adverse events to exercise therapy might be common, but not serious. ${ }^{81}$ Potential adverse reactions to interventions may include muscle soreness or increased pain around the shoulder joint. The physiotherapist will record any adverse reactions to interventions, including duration and severity of adverse reaction to treatment, and how the adverse reaction was managed. We will include in the report the total number of participants who reported adverse events, relatedness to interventions, the duration and severity of the adverse reactions. In the small sample of this feasibility trial, we do not expect to observe a representative number of adverse events, so do not intend statistical comparisons; rather, we will assess the feasibility of the recording forms and systems for use in the main trial.

\section{Health outcomes}

Health outcomes will be expressed as quality-adjusted life years (QALYs) using the Short-Form 12 (SF-12v2) ${ }^{82}$ The SF-12v2 will be converted to a six-dimensional health state classification (SF-6D) ${ }^{83}$ The SF-6D allows estimating the QALY. A QALY is a year of life experienced with a particular health-related quality of life, and will be expressed as a score ranging from 0 to 1 , with $0=$ death and $1=$ full health. Total QALY will be estimated for each participant by calculating the area under the curve (the product of utility values by time). We will calculate the mean QALYs for each group and adjust for baseline utility scores to minimise any bias 


\begin{tabular}{|c|c|c|c|c|c|c|}
\hline & \multicolumn{6}{|c|}{ Study Period } \\
\hline & \multirow{2}{*}{$\begin{array}{l}\text { Enrolment } \\
-\mathrm{t} 1\end{array}$} & \multirow{2}{*}{$\begin{array}{l}\text { Allocation } \\
0\end{array}$} & \multicolumn{4}{|c|}{ Post allocation } \\
\hline Time point & & & Baseline & $\begin{array}{l}4 \\
\text { weeks }\end{array}$ & $\begin{array}{l}8 \\
\text { weeks }\end{array}$ & $\begin{array}{l}12 \\
\text { weeks }\end{array}$ \\
\hline \multicolumn{7}{|l|}{ Enrolment } \\
\hline Eligibility screen & $\mathrm{X}$ & & & & & \\
\hline Informed consent & $\mathrm{X}$ & & & & & \\
\hline \multicolumn{7}{|l|}{ Allocation } \\
\hline \multicolumn{7}{|l|}{ Intervention } \\
\hline \multicolumn{7}{|l|}{ Standardized rehabilitation } \\
\hline \multicolumn{7}{|l|}{ Tailored rehabilitation } \\
\hline \multicolumn{7}{|l|}{ Assessments } \\
\hline Baseline Demographic information & $\mathrm{X}$ & & & & & \\
\hline \multicolumn{7}{|l|}{ Covariates: } \\
\hline Age & & & $\mathrm{X}$ & & & \\
\hline Height & & & $\mathrm{X}$ & & & \\
\hline Weight & & & $\mathrm{X}$ & & & \\
\hline \multicolumn{7}{|l|}{$\begin{array}{l}\text { Primary, and secondary outcome } \\
\text { measures: }\end{array}$} \\
\hline Recruitment rates & & & $\mathrm{X}$ & $\mathrm{X}$ & $\mathrm{X}$ & $\mathrm{X}$ \\
\hline $\begin{array}{l}\text { Adherence to the rehabilitation } \\
\text { programme }\end{array}$ & & & $\mathrm{X}$ & $\mathrm{X}$ & $\mathrm{X}$ & $\mathrm{X}$ \\
\hline $\begin{array}{l}\text { Proportion of participants } \\
\text { enrolled }\end{array}$ & & & $\mathrm{X}$ & $\mathrm{X}$ & $\mathrm{X}$ & $\mathrm{X}$ \\
\hline Drop-out rate & & & $\mathrm{X}$ & $\mathrm{X}$ & $\mathrm{X}$ & $\mathrm{X}$ \\
\hline Adverse reaction & & & $\mathrm{X}$ & $\mathrm{X}$ & $\mathrm{X}$ & $\mathrm{X}$ \\
\hline Pain intensity & & & $\mathrm{X}$ & $\mathrm{X}$ & $\mathrm{X}$ & $\mathrm{X}$ \\
\hline \multicolumn{7}{|l|}{ Health outcomes } \\
\hline Patient specific functional scale & & & $\mathrm{X}$ & $\mathrm{X}$ & $\mathrm{X}$ & $\mathrm{X}$ \\
\hline SPADI & & & $\mathrm{X}$ & $\mathrm{X}$ & $\mathrm{X}$ & $\mathrm{X}$ \\
\hline OCC-Q-Shoulder & & & $\mathrm{X}$ & & & $\mathrm{X}$ \\
\hline Pain self-efficacy questionnaire & & & $\mathrm{X}$ & $\mathrm{X}$ & $\mathrm{X}$ & $\mathrm{X}$ \\
\hline
\end{tabular}

Figure 2 Schedule for enrolment and intervention per group. OCC-Q-Shoulder, Otago Cost and Consequences QuestionnaireShoulder; SPADI, Shoulder Pain and Disability Index.

due to chance baseline imbalance between the groups. As this feasibility trial is not powered to detect superiority, we will assess the magnitude of mean effect on QALYs in relation to clinically important change.

We will adapt the Otago Cost and Consequences Questionnaire (OCC-Q) to shoulder disorders, and use the adapted questionnaire to capture healthcare use and costs for participants enrolled in this study. ${ }^{84}$ The OCC-Q is a patient-administered questionnaire developed for osteoarthritis that has demonstrated accuracy and agreement with administrative databases from the national healthcare system in NZ. ${ }^{84}$ The OCC-Q will be administered at baseline and 12-week time points.

\section{Time points}

Outcome measures will be recorded at baseline, 4th, 8th and 12th week after baseline.

\section{Missing data}

When assessing secondary outcome measures, we will use a linear mixed-effect model to compare groups. This method can handle missing data. For the other analysis, in case of missing data, we will assess its distribution to confirm the assumption that data was missed at random. If missing at random is confirmed, we will perform multiple imputations.

\section{STATISTICAL ANALYSIS}

All statistical analyses will be performed using $\mathrm{R}$ software. ${ }^{85}$ Descriptive statistics will be used to analyse: (1) recruitment rates, (2) adherence to the rehabilitation programme, (3) proportion of participants enrolled from the total number screened, (4) drop-out rates, (5) adverse reactions.

We will use a linear mixed-effect model to obtain estimates of intervention effects, we will compare changes in pain and shoulder-related disability scores between the two intervention groups (ie, tailored and standardised rehabilitation). Group intervention (tailored and standardised rehabilitation) will be considered as between-subject factor, 
and 'time-point' (baseline, 4th, 8th and 12th week) will be considered as within-subject factor. Baseline measurements will be considered as covariates. We will conduct an independent linear mixed-effect model for each outcome measure (ie, pain levels and shoulder-related disability). Given this is a feasibility trial, our goal is not to perform hypothesis testing, but rather to perform these analyses as preliminary assessment of any trend in between-group comparisons. This statistical approach is considered appropriate for feasibility or exploratory studies. ${ }^{86}$

To help informing whether or not it is worthwhile conducting the full trial, it is recommended that preliminary between-group comparisons to be performed at the feasibility trial stage. ${ }^{8788}$ For that, CI ranges other than $95 \%$ should be used (eg, $85 \%$ or $75 \%$ CI in addition to the mean difference estimate) when assessing differences between groups from feasibility trials. ${ }^{87}$ For the purposes of this study, we will consider $75 \%$ CI as the probability threshold. ${ }^{87}$ The mean difference between tailored rehabilitation and standardised rehabilitation will need to be larger than the minimum clinically important difference for either pain or shoulder-related disability. Therefore, if we can be, at least, $75 \%$ sure that one arm is superior to the other arm at 3-month follow-up, then we will consider to have sufficient preliminary evidence of a treatment difference. ${ }^{87}$ Such information will be taken into account when assessing whether or not to conduct the full trial. ${ }^{8788}$

\section{ECONOMIC EVALUATION}

We will use an incremental cost-utility analysis, following intention-to-treat principle, to assess differences in costs and utilities between tailored rehabilitation and standardised exercise, and report incremental net monetary benefit. We will use both a health system and a societal perspective to define and measure costs, as is recommended for cost-effectiveness studies. ${ }^{89}$ We will also report the cost-efficacy and direct medical costs within the NZ healthcare system, and will calculate Bayesian credibility intervals (Bayesian analogue of 95\% CIs) to account for uncertainty in measurements due to sampling random variability, ${ }^{90}$ and will plot cost-effectiveness acceptability curves. ${ }^{91}$

\section{NESTED QUALITATIVE STUDY}

We will include a nested qualitative study that will assess participants' experiences about the trial, and will use a thematic analysis to interpret the data. ${ }^{92}$ We will invite all participants to take part in a semi-structured interview. The goal is to gather data about participants' experience in the trial, in particular about the difficulties and barriers faced by participants, the perceived value and positive aspects of the study and any other issue that may arise during the interviews. Findings from the nested qualitative study will add significant value into how we can minimise perceived barriers, and increase adherence with the trial. Interviews will take place once participants complete the intervention programme (ie, at the 8-week follow-up).
We will use thematic qualitative analysis to analyse and interpret transcriptions from interviews with participants. We will conduct the thematic qualitative analysis in five steps: (1) generation of codes (2) identification of themes, (3) revision of themes, (4) definition and naming of themes, (5) interpretation of findings. ${ }^{92}$ We will keep an audit trail, and members of the research team will crosscheck the coding and interpretation of data. We will adopt an iterative approach during data analysis, and will discuss and reflect on each step to identify any inconsistencies during the thematic qualitative analysis. We will analyse, write and submit for publication the qualitative study as a separate manuscript.

\section{NESTED PROCESS EVALUATION STUDY}

We will conduct a process evaluation study using a mixedmethod design. As part of the process evaluation, we will assess the fidelity, dose and reach of interventions implemented during the Otago MASTER Feasibility trial. We will assess fidelity of interventions by monitoring clinical notes, fortnightly, during the intervention period. Two clinical researchers (the PI and a research assistant) will be responsible for conducting the process evaluation. As per the Medical Research Council (MRC; UK) guidelines, ${ }^{21}$ we will adopt an active role, and provide feedback and additional training to clinicians if required. This approach will optimise fidelity of interventions. According to the MRC (UK) guidelines, this is the most appropriate approach for process evaluation during feasibility trials.

We will conduct a focus-group with clinicians once $70 \%$ of data collection has been completed. The focus-group will allow us to assess clinicians' perspectives (barriers and facilitators) about the interventions and the trial. We will use thematic qualitative analysis to analyse and interpret transcriptions from the focus-group. We will conduct the thematic qualitative analysis in five steps: (1) generation of codes, (2) identification of themes, (3) revision of themes, (4) definition and naming of themes, (5) interpretation of findings. ${ }^{92}$ We will keep an audit trail, and members of the research team will crosscheck the coding and interpretation of data. We will adopt an iterative approach during data analysis, and will discuss and reflect on each step to identify any inconsistencies during the thematic qualitative analysis. We will analyse, write and submit for publication the process evaluation study as a separate manuscript.

\section{DISCUSSION}

In the short-term, the impact of this phase II trial is inform whether or not it is feasible to conduct the full efficacy trial. If the full trial is feasible, the medium-term impact of this proposal will be to determine which exercise therapy intervention is superior for managing patients with shoulder subacromial pain.

This protocol has limitations. We did not include in the initial plan a control arm, to optimise costs at phase II, 
and given that some reviews and guidelines suggest exercise therapy as the treatment of choice. ${ }^{101156} \mathrm{~A}$ recent Cochrane Review recommends future trials to compare exercise therapy interventions with placebo. ${ }^{9}$ In addition, during the peer-review process of this protocol, one reviewer raised the relevance of including a placebo $\mathrm{arm}$. If findings from this feasibility trial suggest that it is feasible to conduct a full trial, we will design a three-arm intervention trial to address recommendations from the Cochrane Review. The full trial will consist of: (1) placebo, (2) tailored rehabilitation, (3) standardised rehabilitation. For this feasibility trial, we estimated sample size based on a two-arm intervention. The advantage of this approach is to reduce the required sample size for the feasibility phase, however, it increases uncertainty on estimates of treatment effects. ${ }^{62}$ This will impact on sample size estimation of the full trial, if findings from this trial supports the next trial stage. ${ }^{62}$ To address that uncertainty on treatment effect estimates, we will take this into account when preparing the future trial.

\section{DATA MANAGEMENT}

Data will be collected by trained researchers, using hard copies of forms and questionnaires. These will be safely stored and locked in a filing cabinet based at the Centre for Health, Activity and Rehabilitation Research (CHARR), School of Physiotherapy-University of Otago. A research assistant will enter the data into a Microsoft Excel file, and only the research team will have access to that file. All trial documents will refer to participants with a unique ID (not by name). We will use single-data entry, with $10 \%$ of the data being entered independently by two research assistants and crosschecked. In addition, we will use histograms, stem and leaf plots, clinical and datadriven range checks as part of quality control. ${ }^{93} 94$

\section{TRIAL MONITORING}

The Health Research Council (HRC) Data Monitoring Core Committee (New Zealand) categorised this trial as low risk, and recommended that an independent Data Monitoring Committee was not necessary. The HRC Data Monitoring Core Committee recommended that an internal monitoring process is sufficient to monitor and oversee this trial. Based on these recommendations, the Data Monitoring Committee from the 'Centre for Health, Activity and Rehabilitation Research' (School of Physiotherapy-University of Otago) will monitor and oversee the trial. The research team has opted not to undertake interim analysis.

\section{DECLARATIONS}

\section{Patient and public involvement}

Patients and or public were not involved. Results of this study will be disseminated to study participants by inviting them to join an open-seminar in which the results of the study will be presented. In addition, we will prepare a short report with the main findings of the study and distribute this by email to participants. The burden of the intervention will be assessed by participants through the nested qualitative study. In that study, we will participants' experiences and perceptions about the trial.

\section{Data collection, storage and sharing}

We will store participants' data on a secure local server, and will use unique identification number on follow-up questionnaires. To protect participants' privacy, all identifying information will be stored separately, and deleted following the conclusion of the trial. We will not share or report identifying information. The datasets generated during the study will be available from the corresponding author on reasonable request.

\section{Confidentiality}

The research team will have access to personal information. We will use group mean data to present findings from the study. This will protect confidentiality before, during and after the trial.

\section{Adverse event management}

The risk of a serious adverse event related to the intervention is minimal. If a participant presents with an adverse event, the primary investigator will report it to the internal Data Monitoring Committee (Centre for Health, Activity and Rehabilitation Research-University of Otago) to assess whether it is necessary to report the adverse event to the trial sponsor, and Ethics Committee. We will suspend the trial if more than one serious adverse event of any kind occurs and these are related or caused by the interventions from the trial. If the cause of the events cannot be determined or remediated, and is plausibly related to the intervention, we will terminate the trial.

\section{Protocol amendments}

We will report any protocol change that may benefit participants, impact on participant's safety or that is likely to impact on the outcomes of the study (e.g. study objectives and/or design changes, sample size, study procedures or significant administrative changes).

Acknowledgements We thank Mr Andrew Gray for statistical advice and the financial support from the Health Research Council New Zealand. We also thank Physiotec for permitting us to use exercise images from their exercise database (https://www.physiotec.ca/index.php).

Contributors DCR and ZJT conceived the research question. DCR was responsible for the design of the trial, and is the guarantor. ZJT and GS contributed to the design of interventions. JHA provided guidance on the design the trial and economic analysis. DCR led efforts for securing funding, with the contributions from ZJT, GS and JHA. All authors revised and approved the protocol for the study. All authors revised the manuscript for important content and approved the final version.

Funding This work is supported by Health Research Council New Zealand (Grant number: 17/536). The trial sponsor is the University of Otago. The trial started in August 2017, and is funded until July 2019. This research was conducted during tenure of The Sir Charles Hercus Health Research Fellowship of the Health Research Council of New Zealand (Grant number: 18/111) awarded to DCR. The Health Research Council - New Zealand had no role in the design of the trial and will have 
no role in its execution, data analysis and interpretation, or on the submission of the studies for publication.

Competing interests None declared.

Patient consent for publication Not required

Ethics approval University of Otago Ethics Committee (Ref: H17/080).

Provenance and peer review Not commissioned; externally peer reviewed.

Open access This is an open access article distributed in accordance with the Creative Commons Attribution Non Commercial (CC BY-NC 4.0) license, which permits others to distribute, remix, adapt, build upon this work non-commercially, and license their derivative works on different terms, provided the original work is properly cited, appropriate credit is given, any changes made indicated, and the use is non-commercial. See: http://creativecommons.org/licenses/by-nc/4.0/.

\section{REFERENCES}

1. Svebak S, Hagen K, Zwart J-A. One-year prevalence of chronic musculoskeletal pain in a large adult Norwegian County population: relations with age and gender -The HUNT Study. J Musculoskelet Pain 2006;14:21-8.

2. Virta L, Joranger P, Brox Jl, et al. Costs of shoulder pain and resource use in primary health care: a cost-of-illness study in Sweden. BMC Musculoskelet Disord 2012;13:17.

3. ACC. Injury statistics tool 2016. Available: http://www.acc.co.nz/ about-acc/statistics/index.htm [Accessed 11 Aug 2016]

4. Bonde JP, Mikkelsen S, Andersen JH, et al. Prognosis of shoulder tendonitis in repetitive work: a follow up study in a cohort of Danish industrial and service workers. Occup Environ Med 2003;60:8e-8.

5. Struyf F, Geraets J, Noten S, et al. A multivariable prediction model for the Chronification of non-traumatic shoulder pain: a systematic review. Pain Physician 2016;19:1-10.

6. British Elbow and Shoulder Society BOA RCoS. Commissioning guide: Subacromial Shoulder Pain. In: Commissioning guide: subacromial shoulder pain. British Elbow \& Shoulder Society (BESS) BOAB, Royal College of Surgeons for England (RCSEng), ed., 2014.

7. Hanratty CE, McVeigh JG, Kerr DP, et al. The effectiveness of physiotherapy exercises in subacromial impingement syndrome: a systematic review and meta-analysis. Semin Arthritis Rheum 2012;42:297-316.

8. Kromer TO, Tautenhahn UG, de Bie RA, et al. Effects of physiotherapy in patients with shoulder impingement syndrome: a systematic review of the literature. J Rehabil Med 2009;41:870-80.

9. Page MJ, Green S, McBain B, et al. Manual therapy and exercise for rotator cuff disease. Cochrane Database Syst Rev 2016;1.

10. Steuri R, Sattelmayer M, Elsig S, et al. Effectiveness of conservative interventions including exercise, manual therapy and medical management in adults with shoulder impingement: a systematic review and meta-analysis of RCTs. Br J Sports Med 2017;51:1340-7.

11. Gebremariam L, Hay EM, van der Sande R, et al. Subacromial impingement syndrome--effectiveness of physiotherapy and manual therapy. Br J Sports Med 2014;48:1202-8.

12. Littlewood C, Ashton J, Chance-Larsen K, et al. Exercise for rotator cuff tendinopathy: a systematic review. Physiotherapy 2012;98:101-9.

13. Littlewood C, May S, Walters S. A review of systematic reviews of the effectiveness of conservative interventions for rotator cuff tendinopathy. Shoulder Elbow 2013;5:151-67.

14. Ho C-YC, Sole G, Munn J. The effectiveness of manual therapy in the management of musculoskeletal disorders of the shoulder: a systematic review. Man Ther 2009;14:463-74.

15. Bang MD, Deyle GD. Comparison of supervised exercise with and without manual physical therapy for patients with shoulder impingement syndrome. J Orthop Sports Phys Ther 2000;30:126-37.

16. Conroy DE, Hayes KW. The effect of joint mobilization as a component of comprehensive treatment for primary shoulder impingement syndrome. J Orthop Sports Phys Ther 1998;28:3-14.

17. Senbursa G, Baltaci G, Atay A. Comparison of conservative treatment with and without manual physical therapy for patients with shoulder impingement syndrome: a prospective, randomized clinical trial. Knee Surg Sports Traumatol Arthrosc 2007;15:915-21.

18. Littlewood C, Malliaras P, Mawson S, et al. Self-managed loaded exercise versus usual physiotherapy treatment for rotator cuff tendinopathy: a pilot randomised controlled trial. Physiotherapy 2014;100:54-60

19. Ingwersen KG, Christensen R, Sørensen L, et al. Progressive highload strength training compared with general low-load exercises in patients with rotator cuff tendinopathy: study protocol for a randomised controlled trial. Trials 2015;16:27.
20. Struyf F, Nijs J, Mollekens S, et al. Scapular-focused treatment in patients with shoulder impingement syndrome: a randomized clinical trial. Clin Rheumatol 2013;32:73-85.

21. Moore GF, Audrey S, Barker M, et al. Process evaluation of complex interventions: medical Research Council guidance. BMJ 2015;350.

22. Oakley A, Strange V, Bonell C, et al. Process evaluation in randomised controlled trials of complex interventions. BMJ 2006;332:413-6.

23. Mars T, Ellard D, Carnes D, et al. Fidelity in complex behaviour change interventions: a standardised approach to evaluate intervention integrity. BMJ Open 2013;3:e003555.

24. Toomey E, Matthews J, Hurley DA. Using mixed methods to assess fidelity of delivery and its influencing factors in a complex selfmanagement intervention for people with osteoarthritis and low back pain. BMJ Open 2017;7:e015452.

25. Linnan L, Steckler A. Process evaluation for public health interventions and research: an overview. In: Steckler A, Linnan L, eds. Process evaluation for public health interventions and research. San Francisco, CA: Jossey-Bass, 2002: 1-23.

26. Kromer TO, Sieben JM, de Bie RA, et al. Influence of fear-avoidance beliefs on disability in patients with subacromial shoulder pain in primary care: a secondary analysis. Phys Ther 2014;94:1775-84.

27. Lentz TA, Barabas JA, Day T, et al. The relationship of pain intensity, physical impairment, and pain-related fear to function in patients with shoulder pathology. J Orthop Sports Phys Ther 2009;39:270-7.

28. Chester R, Smith TO, Hooper L, et al. The impact of subacromial impingement syndrome on muscle activity patterns of the shoulder complex: a systematic review of electromyographic studies. BMC Musculoskelet Disord 2010;11:45.

29. Martinez-Calderon J, Struyf F, Meeus M, et al. The association between pain beliefs and pain intensity and/or disability in people with shoulder pain: a systematic review. Musculoskelet Sci Pract 2018;37:29-57.

30. Chester R, Jerosch-Herold C, Lewis J, et al. Psychological factors are associated with the outcome of physiotherapy for people with shoulder pain: a multicentre longitudinal cohort study. $\mathrm{Br} J$ Sports Med 2018;52:269-75.

31. Ellenbecker TS, Cools A. Rehabilitation of shoulder impingement syndrome and rotator cuff injuries: an evidence-based review. $\mathrm{Br} J$ Sports Med 2010;44:319-27.

32. Moen MH, de Vos R-J, Ellenbecker TS, et al. Clinical tests in shoulder examination: how to perform them. Br J Sports Med 2010;44:370-5.

33. Hegedus EJ, Goode AP, Cook CE, et al. Which physical examination tests provide clinicians with the most value when examining the shoulder? update of a systematic review with meta-analysis of individual tests. Br J Sports Med 2012;46:964-78.

34. Lewis J, McCreesh K, Roy J-S, et al. Rotator cuff tendinopathy: Navigating the Diagnosis-Management conundrum. J Orthop Sports Phys Ther 2015;45:923-37.

35. Reijneveld EAE, Noten S, Michener LA, et al. Clinical outcomes of a scapular-focused treatment in patients with subacromial pain syndrome: a systematic review. Br J Sports Med 2017;51:436-41.

36. Kibler WB, Ludewig PM, McClure PW, et al. Clinical implications of scapular dyskinesis in shoulder injury: the 2013 consensus statement from the 'Scapular Summit'. Br J Sports Med 2013;47:877-85.

37. Hickey D, Solvig V, Cavalheri V, et al. Scapular dyskinesis increases the risk of future shoulder pain by $43 \%$ in asymptomatic athletes: a systematic review and meta-analysis. Br J Sports Med 2018:52:102-10.

38. Spall P, Ribeiro DC, Sole G. Electromyographic activity of shoulder girdle muscles in patients with symptomatic and asymptomatic rotator cuff tears: a systematic review and meta-analysis. PM $R$ 2016;8:894-906.

39. Michener LA, Sharma S, Cools AM, et al. Relative scapular muscle activity ratios are altered in subacromial pain syndrome. J Shoulder Elbow Surg 2016;25:1861-7.

40. Cools AMJ, Struyf F, De Mey K, et al. Rehabilitation of scapular dyskinesis: from the office worker to the elite overhead athlete. $\mathrm{Br} J$ Sports Med 2014;48:692-7.

41. Teys P, Bisset L, Vicenzino B. The initial effects of a Mulligan's mobilization with movement technique on range of movement and pressure pain threshold in pain-limited shoulders. Man Ther 2008;13:37-42

42. Bisset L, Beller E, Jull G, et al. Mobilisation with movement and exercise, corticosteroid injection, or wait and see for tennis elbow: randomised trial. BMJ 2006;333.

43. Ribeiro DC, Castro MPde, Sole G, et al. The initial effects of a sustained glenohumeral postero-lateral Glide during elevation on shoulder muscle activity: a repeated measures study on asymptomatic shoulders. Man Ther 2016;22:101-8. 
44. Ribeiro DC, Sole G, Venkat R, et al. Differences between clinicianand self-administered shoulder sustained mobilization on scapular and shoulder muscle activity during shoulder abduction: a repeatedmeasures study on asymptomatic individuals. Musculoskelet Sci Pract 2017;30:25-33.

45. Ribeiro DC, Sole G, Shemmell J, et al. The initial effects on shoulder muscle activity of shoulder mobilization with movement during shoulder abduction: a repeated-measures study on patients with pain-limited shoulder elevation. ISEK 2018 - XXII Congress of the International Society of Electrophysiology and Kinesiology. Dublin Int Soc Electrophysiol Kinesiol 2018:245-6.

46. Hoyek N, Di Rienzo F, Collet C, et al. The therapeutic role of motor imagery on the functional rehabilitation of a stage II shoulder impingement syndrome. Disabil Rehabil 2014;36:1113-9.

47. Savoie A, Mercier C, Desmeules F, et al. Effects of a movement training oriented rehabilitation program on symptoms, functional limitations and acromiohumeral distance in individuals with subacromial pain syndrome. Man Ther 2015;20:703-8.

48. Worsley P, Warner M, Mottram S, et al. Motor control retraining exercises for shoulder impingement: effects on function, muscle activation, and biomechanics in young adults. $J$ Shoulder Elbow Surg 2013;22:e11-19.

49. Roy J-S, Moffet H, Hébert LJ, et al. Effect of motor control and strengthening exercises on shoulder function in persons with impingement syndrome: a single-subject study design. Man Ther 2009;14:180-8.

50. Singal AG, Higgins PDR, Waljee AK. A primer on effectiveness and efficacy trials. Clin Trans/ Gastroenterol 2014;5:e45.

51. Drummond M, Coyle D. The role of pilot studies in the economic evaluation of health technologies. Int J Technol Assess Health Care 1998;14:405-18.

52. Williams I, Bryan S, Mclver S. How should cost-effectiveness analysis be used in health technology coverage decisions? Evidence from the National Institute for health and clinical excellence approach. J Health Serv Res Policy 2007;12:73-9.

53. Chan A-W, Tetzlaff JM, Gøtzsche PC, et al. SPIRIT 2013 explanation and elaboration: guidance for protocols of clinical trials. BMJ 2013;346:e7586.

54. Hoffmann TC, Glasziou PP, Boutron I, et al. Better reporting of interventions: template for intervention description and replication (TIDieR) checklist and guide. BMJ 2014;348.

55. Moher D, Hopewell S, Schulz KF, et al. Consort 2010 explanation and elaboration: updated guidelines for reporting parallel group randomised trials. BMJ 2010;340:c869.

56. Kulkarni R, Gibson J, Brownson P, et al. Subacromial shoulder pain ESS/BOA patient care pathways. Shoulder Elbow 2015;7:135-43.

57. Kelly SM, Brittle N, Allen GM. The value of physical tests for subacromial impingement syndrome: a study of diagnostic accuracy. Clin Rehabil 2010;24:149-58.

58. Satpute KH, Bhandari P, Hall T. Efficacy of hand behind back mobilization with movement for acute shoulder pain and movement impairment: a randomized controlled trial. J Manipulative Physiol Ther 2015;38:324-34.

59. Hermans J, Luime JJ, Meuffels DE, et al. Does this patient with shoulder pain have rotator cuff disease?: the rational clinical examination systematic review. JAMA 2013;310:837-47.

60. Abbott $\mathrm{JH}$. The distinction between randomized clinical trials (RCTs) and preliminary feasibility and pilot studies: what they are and are not. J Orthop Sports Phys Ther 2014;44:555-8.

61. Shanyinde M, Pickering RM, Weatherall M. Questions asked and answered in pilot and feasibility randomized controlled trials. BMC Med Res Methodol 2011;11:117.

62. Whitehead AL, Julious SA, Cooper CL, et al. Estimating the sample size for a pilot randomised trial to minimise the overall trial sample size for the external pilot and main trial for a continuous outcome variable. Stat Methods Med Res 2016;25:1057-73.

63. Heald SL, Riddle DL, Lamb RL. The shoulder pain and disability index: the construct validity and responsiveness of a region-specific disability measure. Phys Ther 1997;77:1079-89.

64. Roddy E, Zwierska I, Hay EM, et al. Subacromial impingement syndrome and pain: protocol for a randomised controlled trial of exercise and corticosteroid injection (the support trial). BMC Musculoskelet Disord 2014;15:81.

65. Wang SS, Trudelle-Jackson EJ. Comparison of customized versus standard exercises in rehabilitation of shoulder disorders. Clin Rehabil 2006;20:675-85.

66. Borg GA. Psychophysical bases of perceived exertion. Med Sci Sports Exerc 1982;14:377-81.

67. McGuigan MR, Egan AD, Foster C. Salivary cortisol responses and perceived exertion during high intensity and low intensity bouts of resistance exercise. J Sports Sci Med 2004;3:8-15.
68. Heine PJ, Williams MA, Williamson E, et al. Development and delivery of an exercise intervention for rheumatoid arthritis: strengthening and stretching for rheumatoid arthritis of the hand (SARAH) trial. Physiotherapy 2012:98:121-30.

69. Ginn KA, Cohen ML. Exercise therapy for shoulder pain aimed at restoring neuromuscular control: a randomized comparative clinical trial. J Rehabil Med 2005;37:115-22.

70. Teys P, Bisset L, Collins N, et al. One-Week time course of the effects of Mulligan's mobilisation with movement and taping in painful shoulders. Man Ther 2013;18:372-7.

71. Kromer TO, de Bie RA, Bastiaenen CHG. Physiotherapy in patients with clinical signs of shoulder impingement syndrome: a randomized controlled trial. J Rehabil Med 2013;45:488-97.

72. Østerås H, Torstensen TA, Haugerud L, et al. Dose-response effects of graded therapeutic exercises in patients with long-standing subacromial pain. Adv Physiother 2009;11:199-209.

73. Holmgren $\mathrm{T}$, Björnsson Hallgren $\mathrm{H}$, Öberg B, et al. Effect of specific exercise strategy on need for surgery in patients with subacromial impingement syndrome: randomised controlled study. BMJ 2012;344:e787.

74. De Mey K, Danneels L, Cagnie B, et al. Scapular muscle rehabilitation exercises in overhead athletes with impingement symptoms: effect of a 6-week training program on muscle recruitment and functional outcome. Am J Sports Med 2012;40:1906-15.

75. Breivik $\mathrm{H}$, Borchgrevink PC, Allen SM, et al. Assessment of pain. Br J Anaesth 2008;101:17-24.

76. Abbott JH, Schmitt JS. The patient-specific functional scale was valid for group-level change comparisons and between-group discrimination. J Clin Epidemiol 2014:67:681-8.

77. Nicholas MK. The pain self-efficacy questionnaire: taking pain into account. Eur J Pain 2007:11:153-63.

78. Mintken PE, Glynn P, Cleland JA. Psychometric properties of the shortened disabilities of the arm, shoulder, and hand questionnaire (QuickDASH) and numeric pain rating scale in patients with shoulder pain. J Shoulder Elbow Surg 2009;18:920-6.

79. Abbott JH, Schmitt J. Minimum important differences for the patientspecific functional scale, 4 region-specific outcome measures, and the numeric pain rating scale. $J$ Orthop Sports Phys Ther 2014;44:560-4

80. Thabane L, Ma J, Chu R, et al. A tutorial on pilot studies: the what, why and how. BMC Med Res Methodol 2010;10:1.

81. Johnson GM, Skinner MA, Stephen RE. Lessons to be learned: a retrospective analysis of physiotherapy injury claims. J Orthop Sports Phys Ther 2012;42:698-704.

82. Ware J, Kosinski M, Keller SD. A 12-Item short-form health survey: construction of scales and preliminary tests of reliability and validity. Med Care 1996;34:220-33.

83. Brazier JE, Roberts J. The estimation of a preference-based measure of health from the SF-12. Med Care 2004;42:851-9.

84. Pinto D, Robertson MC, Hansen P, et al. Good agreement between questionnaire and administrative databases for health care use and costs in patients with osteoarthritis. BMC Med Res Methodol $2011 ; 11$.

85. R_Core_Team. R: a language and environment for statistical computing. $\mathrm{R}$ foundation for statistical computing, Vienna, Austria. ISBN 3-900051-07-0, 2008. Available: http://www.R-project.org [Accessed 1 Mar 2011].

86. Perneger TV. What's wrong with Bonferroni adjustments. BMJ 1998;316:1236-8.

87. Lee EC, Whitehead AL, Jacques RM, et al. The statistical interpretation of pilot trials: should significance thresholds be reconsidered? BMC Med Res Methodol 2014;14.

88. Julious SA, Tan SB, Machin D. An introduction to statistics in early phase trials. Wiley-Blackwell, 2010: 264.

89. Drummond M, Manca A, Sculpher M. Increasing the generalizability of economic evaluations: recommendations for the design, analysis, and reporting of studies. Int J Technol Assess Health Care 2005;21:165-71.

90. Briggs AH. A Bayesian approach to stochastic cost-effectiveness analysis. An illustration and application to blood pressure control in type 2 diabetes. Int J Technol Assess Health Care 2001;17:69-82.

91. Fenwick E, Claxton K, Sculpher M. Representing uncertainty: the role of cost-effectiveness acceptability curves. Health Econ 2001;10:779-87.

92. Braun V, Clarke V. Using thematic analysis in psychology. Qual Res Psychol 2006;3:77-101.

93. Day S, Fayers P, Harvey D. Double data entry: what value, what price? Control Clin Trials 1998;19:15-24.

94. Büchele G, Och B, Bolte G, et al. Single vs. double data entry. Epidemiology 2005;16:130-1. 
95. Hing W, Hall T, Rivett DA, et al. The Mulligan concept of manual therapy, textbook of techniques. 1st ed. Churchill Livingstone, 2015: 500.

96. Magarey ME, Jones MA. Dynamic evaluation and early management of altered motor control around the shoulder complex. Man Ther 2003;8:195-206.
97. Magarey ME, Jones MA. Specific evaluation of the function of force couples relevant for stabilization of the glenohumeral joint. Man Ther 2003;8:247-53

98. Caldwell C, Sahrmann S, Van Dillen L. Use of a movement system impairment diagnosis for physical therapy in the management of a patient with shoulder pain. J Orthop Sports Phys Ther 2007;37:551-63. 